\title{
Analytical performance modeling of elastic optical links with aligned spectrum allocation
}

\author{
Kaveh Vaezi ${ }^{\mathrm{a}, 1}$, Nail Akar ${ }^{\mathrm{b}, *}$ \\ a Department of Electrical Engineering, Sharif University of Technology, Tehran, Iran \\ ${ }^{\mathrm{b}}$ Electrical and Electronics Engineering Department, Bilkent University, Ankara, Turkey
}

\section{A R T I C L E I N F O}

\section{Article history:}

Received 23 October 2014

Revised 20 April 2015

Accepted 9 June 2015

Available online 16 June 2015

\section{Keywords:}

Elastic optical networks

Spectrum allocation

Markov modulated Poisson process

Bandwidth blocking probability

\begin{abstract}
A B S T R A C T
Elastic optical networking has recently been proposed for use in optical transport networks to cope with increasingly heterogeneous and dynamic demand patterns. In this paper, we study the blocking performance of a multi-class elastic optical link for which a demand needs to be allocated a contiguous subset of the entire spectrum. This problem is different than the well-known blocking problem in multi-class multi-server loss systems due to the contiguous allocation constraint. We first propose a non-work-conserving aligned spectrum allocation policy which is shown to outperform the conventional first fit-based work-conserving allocation policy without alignment. Subsequently, for blocking performance of an aligned elastic optical link with up to three different traffic classes, we propose a novel and systematic order reduction procedure for MMPPs (Markov Modulated Poisson Process) and use this procedure as the numerical engine to approximately obtain the blocking probabilities. The proposed numerical algorithm is validated under various system and traffic parameters and is shown to be effectively usable as an instrument to dimension elastic optical links.
\end{abstract}

(c) 2015 Elsevier B.V. All rights reserved.

\section{Introduction}

In the past several years, the telecommunications industry has witnessed a tremendous increase in the amount of IP traffic driven by more intensive use of video-based communications, increased use of smart phones, increased penetration of wireless/wireline broadband access, etc. This exponential increase in the Internet traffic has been stressing the capacity of carrier optical transport networks. Current state of the art optical transport networks employ Dense Wavelength Division Multiplexed (DWDM) transmission with perwavelength capacities of 10,40 , or $100 \mathrm{Gbps}[1,2]$. Not only the per-wavelength capacities have increased recently, the

\footnotetext{
* Corresponding author. Tel.: +90 312 2902337; fax: +90 3122664192.

E-mail addresses: k.vaezi@gmail.com (K. Vaezi), akar@ee.bilkent.edu.tr (N. Akar).

1 This work is done during the visit of Mr. Vaezi to the Electrical and Electronics Engineering Department, Bilkent University, Ankara, Turkey.
}

reach of the optical signals has expanded significantly making it possible to reduce the Optical-Electrical-Optical (OEO) regeneration costs [3]. Optical cross-connects (OXC) (with or without wavelength conversion capability) have the wavelength switching capability to route the optical signal from one end point to another in DWDM networks, hence referred to as a Wavelength Routed Network (WRN). The path taken by the optical signal in WRNs is called an Optical Path (OP). The International Telecommunication Union (ITU) currently employs a fixed wavelength grid which divides the optical spectrum range at the C-band (1530-1565 nm) into fixed $50 \mathrm{GHz}$ spectrum slots. The following issues have been identified for current DWDM optical transport networks:

(i) Although current $100 \mathrm{Gbps}$ transmission systems are able to use the $50 \mathrm{GHz}$ fixed grid, transmissions beyond $100 \mathrm{Gbps}$, in particular $400 \mathrm{Gbps}$ and $1 \mathrm{Tbps}$ optical signals, can not fit into a fixed $50 \mathrm{GHz}$ slot [2] at standard modulation formats. Therefore there is a need to efficiently accommodate super-wavelength 
traffic, i.e., traffic exceeding the capacity of a wavelength.

(ii) In current WRNs, the entire capacity of a wavelength needs to be allocated to an OP. This leads to very inefficient use of fiber resources in case the traffic using the optical path is not able to fill the pipe which therefore gives rise to a need to accommodate sub-wavelength traffic.

(iii) Besides the granularity mismatch between the traffic demands and the rigid wavelength capacity, current DWDM transmission systems consider the worst case transmission scenario. Particularly, a modulation format is chosen for an optical signal with the assumption that it will traverse the most challenging path in terms of distance, number of OXCs, number of repeaters, etc., and this format is then used for all such signal transmissions irrespective of the path they will traverse. However, modulation formats with larger constellation sizes may be preferred for less challenging shorter-reach paths in order to increase the spectral efficiency.

(iv) Current WRNs possess a rigid bandwidth allocation which is not suitable for time-varying traffic in terms of bandwidth and power efficiency. There is a need to expand and shrink optical resources with respect to time-varying characteristics of client IP traffic for power and bandwidth efficiency purposes.

(v) Fixed grids employ relatively large guard bands between optical paths. There is a need to reduce bandwidth waste stemming from guard bands.

The issues described above related to the need for more efficient bandwidth sharing, are addressed by packet switching-based optical technologies, for example Optical Packet Switching (OPS) and Optical Burst Switching (OBS) [4-6]. However, such technologies are generally viewed as longer term solutions due to the lack of practical optical buffers and high costs of wavelength converters that are needed for acceptable bandwidth efficiency in OPS or OBS.

A recent paradigm, called Elastic Optical Networks (EON), has recently emerged as a short-term solution addressing the issues that DWDM-based WRNs raise [2,3]. EONs rely on the flexgrid scheme where the available optical spectrum is divided into frequency slots that have finer spectral width compared to the $50 \mathrm{GHz}$ ITU-T frequency grid. Potential alternatives for the finer Slot Width (SW) are $6.25 \mathrm{GHz}, 12.5 \mathrm{GHz}$, or $25 \mathrm{GHz}$ [7]. With the use of Liquid Crystal on Silicon (LCOS) devices, adjacent slots can be joined together to form a spectral block that can be dedicated to a single OP [8]. Moreover, different modulation formats, as opposed to a single standard one, can be used for different OPs in EONs. EONs have been demonstrated in the SLICE network detailed in [3] using Orthogonal Frequency Division Multiplexing (OFDM). For a recent review of EONs, we refer the reader to [9].

Demands in EONs are classified into several classes on the basis of their spectral requirements, hence referred to as a multi-class EON. In a $K$-class EON, a class- $k$ demand, $k=0,1, \ldots, K-1$, requires a spectral block of $n_{k} \geq 1$ contiguous frequency slots (or slots in short). Therefore, we say the size of the class- $k$ demand is $n_{k}$. For example, assuming $12.50 \mathrm{GHz}$ slot width, a $40 \mathrm{Gbps}$ (100 Gbps, $400 \mathrm{Gbps}$ ) demand requires a spectral block comprising 2 slots ( 4 slots, 16 slots) using QPSK modulation [10]. When the modulation format is changed to 16-QAM, then the slot requirements would be halved for all three types of demands. Unlike static EONs in which traffic demands do not change in time, in dynamic EONs, demands (or requests) dynamically arrive at the network, stay in the network for a duration known as demand holding time if they are accepted, and then leave the system. Routing and Spectrum Allocation (RSA) problem in dynamic EONs is involved in finding a route and allocate a free spectral block to be used by the demand [11]. If a free spectral block can not be found, then the demand is said to be blocked. For RSA, it should be noted that the frequency slots dedicated to a particular demand need to be identical for all links along the OP (continuity constraint) and also contiguous in spectrum (contiguity constraint), which is known as the Spectrum Continuity and Contiguity Constraint (SCCC). Fragmentation of optical spectrum is a well-known phenomenon in EONs. A spectral block may not be available on all links of a path for a demand although individual links may have sufficient resources known as the horizontal fragmentation problem [12]. In vertical fragmentation, idle spectral resources on individual links are scattered in spectrum making it hard to find large contiguous spectral blocks to be allocated to demands with larger sizes [12]. Spectrum Allocation (SA) policies have been proposed to combat both types of fragmentation. As representative SA policies, the Random Fit (RF) policy places the request randomly in one of the free spectral blocks [13] and the First-Fit (FF) policy places the incoming request in the first available spectral block starting from the low end of the spectrum [14]. Due to fragmentation, the blocking probability for demands with larger sizes are higher than those with smaller demands [12]. Fragmentation is therefore detrimental to not only overall performance but also to fairness [12]. Spectrum allocation policies have been devised to address the above-mentioned fairness problem [12,15-17].

In this paper, our goal is to develop an analytical performance model by which we can compute the blocking probabilities in a multi-class, multi-server loss system with spectrum contiguity constraints, when the per-class arrival processes are Poisson and demand holding times are exponentially distributed. In this model, the set of servers allocated to a single demand need to be adjacent making it different than the case when the contiguity constraint is absent, the latter problem being one of the most well-known tele-traffic loss models of the circuit switching literature and efficient numerical algorithms are available for calculating blocking probabilities [18-21]. The loss system model with contiguity constraints fits well to a single Elastic Optical Link (EOL) that interconnects two nodes of an underlying EON. The performance modeling of more general EONs comprising a mesh network of nodes interconnected by a number of EOLs is left for future study. Most of the existing studies use simulations for performance assessment of dynamic EONs. Analytical models, on the other hand, are rare. The references [22,23] provide an exact Markov chain model for a single link when FF is used for SA. In these works, the transition matrix is sparse and a Gauss-Seidel iteration method is proposed to solve the Markov chain but it is not possible to solve relatively large systems due to the curse of 
dimensionality and only systems with two classes are presented in the numerical examples. The work in [24] proposes an approximate model to analyze the effect of bandwidth fragmentation on the blocking probability in EONs.

In this paper, we propose an approximate Markov model to solve the per-class blocking probabilities in a single EOL. The particular SA method we propose is non-workconserving and allows demands to be placed only in certain aligned spectral blocks, as opposed to work-conserving SA policies such as FF, hence referred to as aligned SA. We also show by simulations that the aligned SA method we propose outperforms the FF policy in terms of bandwidth blocking probability. Our proposed performance modeling methodology relies on MMPP (Markov Modulated Poisson Process)based model reduction to mitigate the curse of dimensionality problem that arises in exact Markov models as in [22]. Using the proposed method, it is possible to accurately characterize the achievable throughput in relatively large capacity EOLs under spectrum contiguity constraints and with $K \leq$ 3 classes of traffic.

The paper is organized as follows. In Section 2, we present the elastic optical link of interest, the related assumptions, and the problem description. In particular, the aligned SA policy is presented along with numerical examples to validate this policy in Section 2. Section 3 addresses the MMPP order reduction method which is crucial for the development of the approximate stochastic model for aligned SA. In Section 4, we present the approximate Markov chain-based model to find the per-class blocking probabilities which uses MMPP order reduction as its numerical engine. Numerical results are provided for validating the proposed model in Section 5. Finally, Section 6 concludes the paper.

\section{Aligned elastic optical links and problem description}

We envision an Elastic Optical Link (EOL) which is interconnecting two EON nodes and comprises $N$ contiguous frequency slots, indexed from 0 (the frequency slot at the low end of the spectrum) to $N-1$ (the frequency slot at the high end of the spectrum). We have $K$ classes of requests numbered from $k=0$ to $K-1$. A class- $k$ connection requires $n_{k}$ contiguous slots to be allocated to that connection. For the sake of convenience, we assume $n_{0}<n_{1}<\ldots<n_{K-1}$. A Spectral Block (SB) of size $n$ refers to a subset of the entire spectrum comprising the frequency slots $a, a+1, \ldots, a+$ $n-1$ for some $0 \leq a \leq N-n$ and is denoted by [a, $a+n-$ $1]$. Let $\mathbf{n}$ denote the vector of per-class demand sizes, i.e., $\mathbf{n}=\left(n_{0}, n_{1}, \ldots, n_{K-1}\right)$ which is referred to as the demand size vector. For work-conserving SA, if there is a free SB of size $n_{k}$ on the link, then the class- $k$ connection request is accepted and one of such free SBs is allocated to the connection. Otherwise, the connection request is blocked. If there are multiple free SBs that can satisfy the request, an SA policy chooses one of the free SBs so as to optimize a certain performance metric. For example, the FF policy studied for benchmarking in this paper places the incoming request in the first available frequency block starting from the low end of the spectrum. For each class- $k, k=0,1, \ldots, K-1$, demands are assumed to arrive at the EOL according to a Poisson process with intensity $\lambda_{k}$ and the holding time of the accepted demands are assumed to be exponentially distributed with parameter $\mu_{k}$.
The load offered by class- $k$ demands is denoted by $\eta_{k}$ :

$\eta_{k}=\frac{n_{k} \lambda_{k}}{\mu_{k}}$

and the overall load, $\eta$, is

$\eta=\sum_{k=0}^{K-1} \eta_{k}$.

The normalized link load $\rho$ is the ratio of the overall load to capacity:

$\rho=\frac{\eta}{N}$.

The overall bandwidth blocking probability $P_{B}$ is given by the following equation:

$P_{B}=\frac{\sum_{k=0}^{K-1} \lambda_{k} n_{k} P_{k}}{\sum_{k=0}^{K-1} \lambda_{k} n_{k}}$,

where $P_{k}$ denotes the class- $k$ demand blocking probability. Two different traffic mixture models are to be used in the numerical examples of this paper. The EI (Equal Intensity) scenario corresponds to the choice of $\lambda_{k}=\lambda$, for $k=$ $0, \ldots, K-1$ for which all traffic classes have the same arrival rate. The EL (Equal Load) scenario refers to one for which $\eta_{k}=\eta / K$, for $k=0, \ldots, K-1$ and in this case the contribution of the traffic classes to the overall EOL load is identical. Numerical study of other traffic mixtures are left for future study but we note that the proposed stochastic model can be applied to all traffic mixtures.

\subsection{Assumptions}

We impose the following assumptions on this system that will allow us to obtain the performance metrics of interest even for relatively large values of $N$.

(a) We limit our attention to 2- and 3-class EOLs only. Although it is possible to extend the model for $K>3$, we omit the discussion of this more general case due to cumbersome notation.

(b) Defining $n_{K}=N$ and $m_{k}=n_{k+1} / n_{k}, 0 \leq k \leq K-1$, we assume that the quantities $m_{k}, 0 \leq k \leq K-1$ are integervalued. For example, consider an EOL with $6.25 \mathrm{GHz}$-wide slots and QPSK-modulation scheme serving 10, 40, and $100 \mathrm{Gbps}$ demands. Consequently, the demand size vector is $\mathbf{n}=(1,4,8)$. If the slot width is doubled, then the new demand size vector becomes $\mathbf{n}=(1,2,4)$. In both cases, the assumption (b) is satisfied.

(c) We assume $n_{0}=1$ without loss of generality since a system with demand size vector $\mathbf{n}=\left(n_{0}, n_{1}, \ldots, n_{K-1}\right)$ and capacity $N$ is statistically equivalent to one with vector $\mathbf{n}=\left(1, m_{0}, m_{0} m_{1}, \ldots, m_{0} m_{1} \cdots m_{K-2}\right)$ with capacity $N / n_{0}$.

However, with recent advances in optical transmission, there may also be realistic scenarios that would not satisfy the above-mentioned assumptions. For example, a recently reported scenario in [25] employing a demand size vector $\mathbf{n}=(3,5,8,16)$ in an EON of four classes serving demands of 100, 200, 400, and 1000 Gbps, would not satisfy the assumptions (a) and (b). Note that, even when $1000 \mathrm{Gbps}$ demands are excluded, the remaining demand size vector 
$\mathbf{n}=(3,5,8)$ would not satisfy assumption (b). We leave an elaborate exploration of more general demand size vectors, the benefits of alignment in these cases, and computationally efficient stochastic modeling of these scenarios for future work.

\subsection{Aligned spectrum allocation}

In this paper, we envision the use of aligned SA for which only spectral blocks in the range $\left[(i-1) n_{k}, i n_{k}-1\right], 1 \leq i \leq$ $N / n_{k}$, called class- $k$ SBs, are allowed for allocation for new class- $k$ demands. We call the EOL aligned if aligned SA is employed. For aligned SA and upon a class- $k$ demand arrival, the proposed aligned SA policy employs first fit among the class$k$ SBs, first the SB $\left[0, n_{k}-1\right]$, then the SB $\left[n_{k}, 2 n_{k}-1\right]$, and so on, until a free $S B$ is found. If not, the demand would be blocked even when a free spectral block of size $n_{k}$ would be free. At a first glance, alignment may be considered harmful for overall performance since there are fewer class- $k$ SBs $\left(N / n_{k}\right.$ of them) as opposed to $N-n_{k}$ SBs of size $n_{k}$ in the non-aligned EOL and the proposed SA policy is therefore non-work-conserving. However, alignment alleviates vertical fragmentation. To explain this, let us a consider a 2-class EOL with $N=4, \mathbf{n}=(1,2)$. In the non-aligned EOL case, the three SBs [0, 1], [1, 2], [2, 3] are available for class-1 requests whereas the aligned EOL is allowed to use only the two SBs $[0,1]$ and $[2,3]$. When the SB $[1,2]$ is allocated to a class-1 demand in the non-aligned EOL, all further class- 1 requests would be blocked even when the slots 0 and 3 are available. The situation may worsen for a 3-class system. To see this, consider a non-aligned EOL with $N=16, \mathbf{n}=(1,2,8)$. When the SB $[7,8]$ is allocated to a class- 1 demand, all forthcoming class-2 demands would be blocked irrespective of the availability of the other slots since with the spectral slots 7 and 8 being occupied, it is not possible to allocate 8 contiguous slots to class- 2 demands. To further illustrate the potential advantage of alignment, consider a system with $N=8, K=$ 3 , and $\mathbf{n}=(1,2,4)$ and the following scenario for which demands arrive at a completely idle EOL with the following order: class- 1 first, then class- 2 and class- 0 , followed by the departure of the class- 2 demand, and then the arrival of a new class- 0 demand, and subsequently a class- 2 demand. This scenario is illustrated in Fig. 1 for non-aligned and aligned EOL cases. It is clear that the second class-2 demand would be blocked in the non-aligned EOL while the aligned EOL does not block any of the five demands. This outcome stems from reduced vertical fragmentation with alignment. Although the above-mentioned scenarios provide insight on why alignment would be beneficial for overall performance in EOLs, an elaborate analysis is necessary to quantify the gains achievable by the non-work-conserving aligned EOL for various scenarios. For this purpose, we conduct a simulation-based study of an aligned EOL in comparison with a non-aligned EOL in terms of the bandwidth blocking probability $P_{B}$ which is the key performance metric used in this study. We first fix $\rho=0.5$ when the demand size vector $\mathbf{n}=(1,4,8)$ for both aligned and non-aligned EOL cases and we use the EL traffic mixture model. We then plot the bandwidth blocking probability $P_{B}$ in Fig. 2 as a function of the capacity $N$ for both aligned and non-aligned EOL cases. We observe that the non-work-conserving aligned EOL slightly outperforms the (a) non-aligned EOL

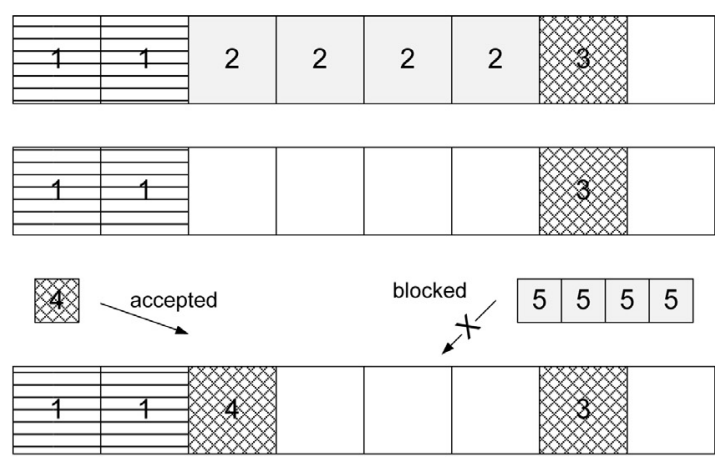

(b) aligned EOL
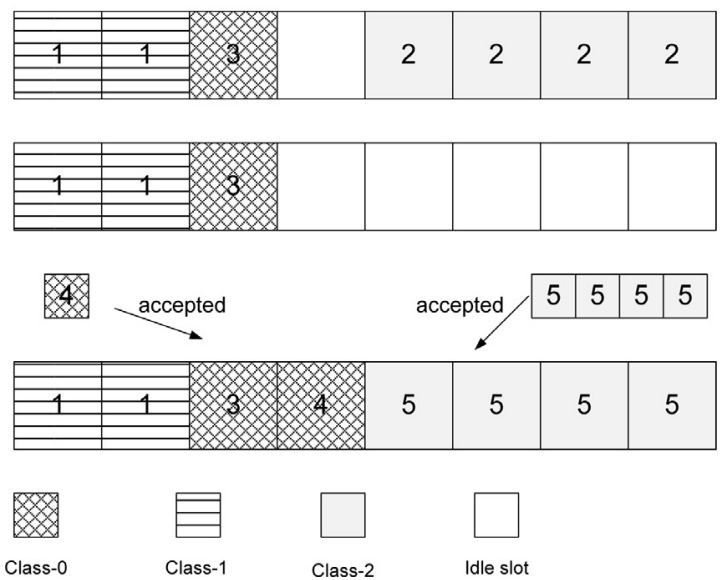

Fig. 1. An example scenario with $N=8, K=3, \mathbf{n}=(1,2,4)$ for which (a) the second class- 2 demand is blocked in the non-aligned EOL (b) all the demands accepted in the aligned EOL.

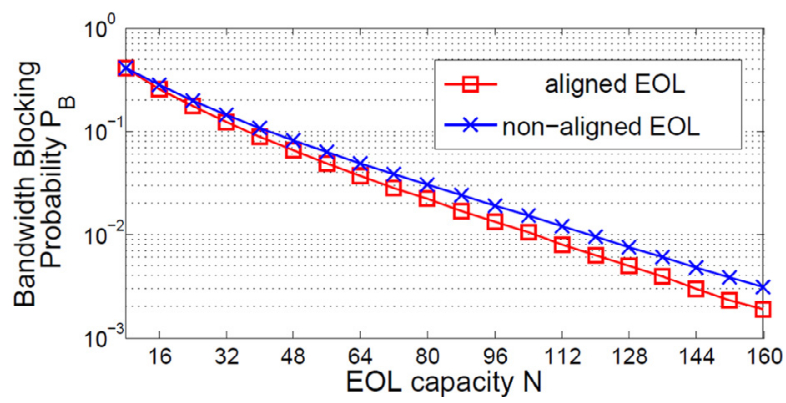

Fig. 2. Bandwidth blocking probability $P_{B}$ for an aligned and non-aligned EOL as a function of the EOL capacity $N$ when $\mathbf{n}=(1,4,8), \rho=0.5$ in the EL mixture scenario.

non-aligned one in terms of $P_{B}$ for all values of $N$ with the performance gap expanding with increased capacity $N$.

In the second numerical example, we study six different scenarios obtained by two different choices of the request size vector, two different values of $\rho$, and the choice of the two EI and EL traffic mixture models. We obtain the percentage improvement (reduction) in $P_{B}$ with alignment in comparison with non-alignment as a function of $N$ for all 


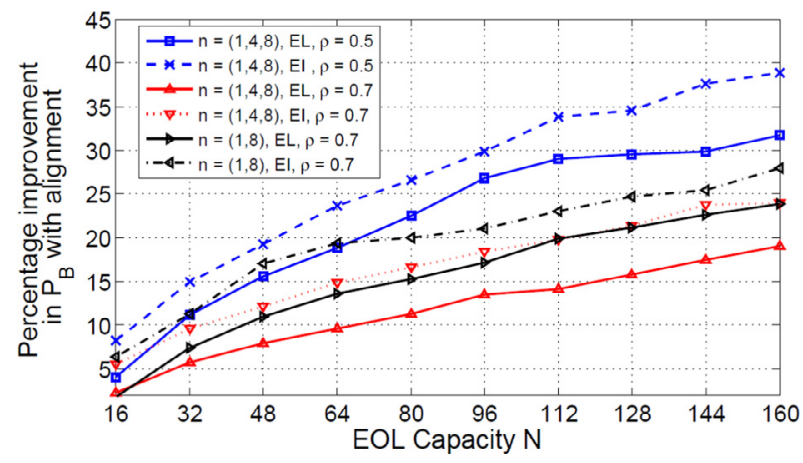

Fig. 3. Percentage improvement in $P_{B}$ with an aligned EOL in comparison with non-aligned EOL for various choices of the demand size vector $\mathbf{n}$ and both EI and EL traffic mixtures.

the six different scenarios in Fig. 3. We observe that alignment consistently reduces the bandwidth blocking probability with the performance improvement increasing with increased system capacity. Moreover, the performance improvement with alignment appears to be more emphasized for lower loads. It is also worth mentioning that besides performance improvement, it is much simpler to implement aligned EOLs since there are far less SBs over which a search would be performed to find the idle SBs. For the rest of the study, we focus only on aligned SA and its stochastic model.

\subsection{Problem description}

The purpose of this paper is to develop a computationally efficient analytical method to numerically obtain the perclass blocking probabilities $P_{k}$ and bandwidth blocking probability $P_{B}$ for an aligned EOL with $K$ classes of traffic with $K \leq 3$ under the traffic and demand size vector assumptions laid out in the previous two subsections. A brute-force approach is to keep track of each frequency slot whether be idle or occupied and if occupied which class of demands is using it [22]. One may then solve a Markov chain of size $(K+1)^{N}$ which is not feasible for large values of $N$. For example, if the entire spectrum is set to $1.6 \mathrm{THz}$ as in [26], then the EOL capacity would be $N=128$ when the slot width is set to $12.5 \mathrm{GHz}$ as in [27]. Consequently, the brute-force approach would fail for any choice of $K \geq 2$. Before describing the proposed stochastic model, a brief review on MMPPs is presented, and a novel MMPP reduction method is proposed which is of key importance for the computational complexity reduction to be attained by the proposed analytical method.

\section{MMPP order reduction method}

The following is based on [28]. A Markov Modulated Poisson Process (MMPP) is a point process whose intensity depends on the state of a background process represented by an irreducible finite-state Continuous-Time Markov Chain (CTMC) with $m$ states for the background process. The continuous-time MMPP is characterized by the infinitesimal generator $Q$ of the modulating CTMC and by its rate matrix $\Lambda$, an $m \times m$ diagonal matrix with diagonal elements $\lambda_{1}, \lambda_{2}, \ldots, \lambda_{m}$, i.e., $\Lambda=\operatorname{diag}\left(\lambda_{1}, \lambda_{2}, \ldots, \lambda_{m}\right)$. For this MMPP characterized by the pair $(Q, \Lambda)$, arrivals occur according to a Poisson process with rate $\lambda_{i}$ when the background process resides at state $i$. The stationary probability vector of the background process is denoted by $\pi$ which satisfies

$\pi Q=0, \quad \pi \mathbf{e}=1$,

where $\mathbf{e}$ is a column vector of ones with appropriate size. The mean arrival rate of the MMPP characterized with the pair $(Q, \Lambda)$ is denoted by $\sigma$ and is given as $\sigma=\pi \Lambda e$ [28] and $m$ represents the order of the MMPP. MMPPs have dependent successive inter-arrival times in contrast with renewal processes which allows them to be used in modeling overflow traffic in circuit-switched networks [29,30]. However, using MMPPs with large state spaces are either computationally infeasible or impractical. Therefore, order reduction is crucial in such cases. Given an original $\operatorname{MMPP}(Q, \Lambda)$ with $m$ states, a reduced order MMPP is one with fewer states $m_{r}<<$ but inherits as many of the statistical features of the original one. For order reduction purposes, we first present a method to aggregate a given subset of states $E_{r}$ of the original MMPP into one single state. The states in the complementary subset $E_{c}$ are preserved. It is clear that $E_{r} \cup E_{c}=E$, which is the state-space of the original MMPP. In the aggregation method, the following conditions need to be satisfied:

(i) The aggregation procedure preserves the average arrival rate.

(ii) The stationary probabilities of the states in $E_{c}$ do not change after aggregation.

(iii) The transition rates among the states in $E_{c}$ are kept unchanged after aggregation.

For this purpose, let us partition $Q, \pi$, and $\Lambda$ as

$Q=\left[\begin{array}{ll}Q_{r r} & Q_{r c} \\ Q_{c r} & Q_{c c}\end{array}\right], \quad \Lambda=\left[\begin{array}{ll}\Lambda_{r} & \\ & \Lambda_{c}\end{array}\right]$,

$\pi=\left[\begin{array}{ll}\pi_{r} & \pi_{c}\end{array}\right]$,

where $Q_{r r}\left(Q_{c c}\right)$ governs the transitions among states in $E_{r}$ $\left(E_{c}\right)$. The reduced order MMPP is characterized by $\left(Q^{r}, \Lambda^{r}\right)$ which are given by the following expressions:

$Q^{r}=\left[\begin{array}{ll}q_{0} & v_{0 c} \\ v_{c 0} & Q_{c c}\end{array}\right], \quad \Lambda^{r}=\left[\begin{array}{ll}\lambda_{0} & \\ & \Lambda_{c}\end{array}\right]$,

$\pi^{r}=\left[\begin{array}{ll}\pi_{0} & \pi_{c}\end{array}\right]$,

where $\pi^{r}$ is the steady-state probability vector of the reduced order MMPP and satisfies

$\pi^{r} Q^{r}=0, \quad \pi^{r} \mathbf{e}=1$

The column vector $v_{c 0}$, the row vector $v_{0 c}$ and the scalars $q_{0}$, $\pi_{0}$ and $\lambda_{0}$ are chosen as follows:

$$
\begin{aligned}
q_{0} & =\frac{\pi_{r} Q_{r r} \mathbf{e}}{\pi_{0}}, \quad \pi_{0}=\pi_{r} \mathbf{e}, \quad \lambda_{0}=\frac{\pi_{r} \Lambda_{r} \mathbf{e}}{\pi_{0}}, \\
v_{0 c} & =-\frac{\pi_{c} Q_{c c}}{\pi_{0}}, \quad v_{c 0}=Q_{c r} \mathbf{e} .
\end{aligned}
$$

It can be shown that with this choice, the three conditions (i)-(iii) stated above are satisfied. Note that if the arrival rates in all the states in $E_{r}$ are identical, then the quantity $\lambda_{0}$ would also be equal to that common rate. In this paper, we refer to this method as the aggregation method. Next, we present the MMPP reduction method we propose in this paper which 
Algorithm 1

Algorithm for obtaining blocking probabilities when $K=2$.

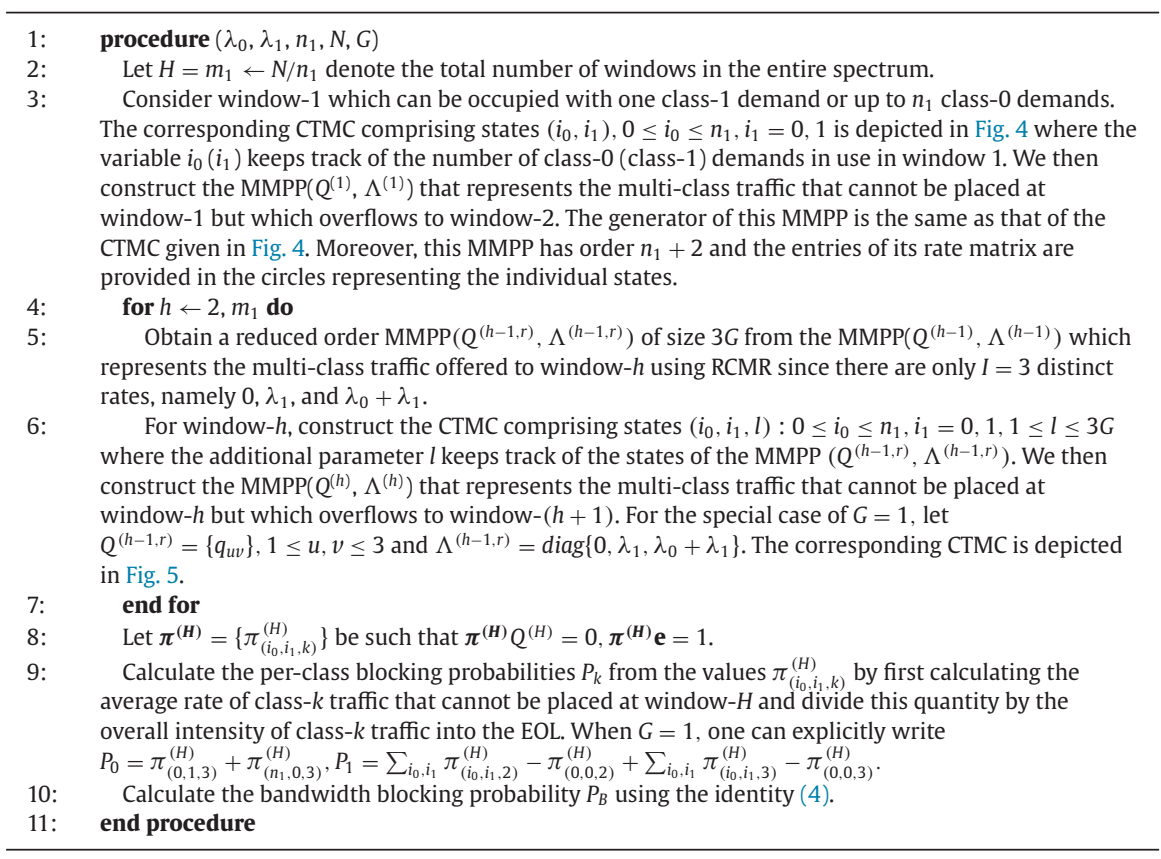

uses the above-mentioned aggregation method as its main building block.

Let us consider the original MMPP $(Q, \Lambda)$. Suppose that there are $I \geq 1$ different subsets of states in the modulating MC such that the states in a given subset have identical arrival rates. Each subset is then partitioned into $G$ sub-subsets in a way that the conditional probability of residence at each of these sub-subsets given that the original MC is visiting a state in the designated subset, is as close as possible to $\frac{1}{G}$. Hence, there are IG sub-subsets of states in the modulating MC of the MMPP and all states in each sub-subset is associated with the same arrival rate. We propose to apply the aforementioned aggregation method IG times to aggregate all the states in one sub-subset into one state to obtain a reduced order MMPP of size $I G$ provided that there are at least $G$ states in a sub-subset. This systematic reduction procedure approximates a large MMPP with a reduced order MMPP with the approximation parameter $G$ establishing a trade-off between the accuracy of this approximation and the computational complexity of the approach. Increasing $G$ results in higher accuracy in the approximation but also increases the number of states in the modulating MC of the reduced MMPP. Note that we also preserve the set of all distinct arrival rates in the model reduction method, hence referred to as RateConserving MMPP Reduction (RCMR) method.

\section{Stochastic model of aligned EOLs}

For the rest of this paper, we refer to the particular $n_{K-1}$ sized SBs as windows. In particular, we have an overall number of $H=m_{K-1}$ windows enumerated from window-1 (the low end of the spectrum) to window- $H$ (the high end of the spectrum). At each step of the proposed algorithm, we consider just one window only. Demands from multiple classes first arrive at the first window. A demand is either accepted at this window or not. In the latter case, it overflows to the second window and checks for free resources in the second window. The procedure is repeated until the demand is either accepted into one of the $H$ windows or is blocked due to lack of any free SBs. For modeling this behavior, we first characterize the overall multi-class traffic overflowing from window-1 to window-2. Although an exact MMPP characterization is possible for this traffic, we propose to use MMPP order reduction method described in Section 2 to reduce the order of this MMPP to a much smaller one to cope with increased computational complexity. We then repeat this procedure for all the $H$ windows and write down the per-class blocking probabilities at the final step from the steady-state behavior of the stochastic process underlying window- $H$. We present the step-by-step algorithm to calculate the blocking probabilities in the specific system $K=2$ illustrated in Algorithm 1. We then describe how the same algorithm can be used in the more general case $K>2$.

Let us now consider the computational complexity of Algorithm 1 for $K=2$ which runs in $H=m_{1}$ steps at each of which the steady-state solution of a CTMC with $3 G\left(n_{1}+2\right)$ states (except for the first step with $\left(n_{1}+2\right)$ states only) needs to be obtained. Therefore, the imposed computational complexity is $\mathcal{O}\left(G^{3} m_{0}^{3} m_{1}\right)$ which is remarkably reduced compared to $\mathcal{O}\left((K+1)^{3 N}\right)=\mathcal{O}\left(27^{m_{1} m_{0}}\right)$. Moreover, one may note that the generator of the CTMC obtained at each step is in the form of a non-homogeneous (or level-dependent) finite Quasi-Birth-and-Death (QBD) process [31,32]. We note that the Linear Level Reduction (LLR) approach of [33] can be used to determine the stationary distribution of the arising CTMC at each step. 


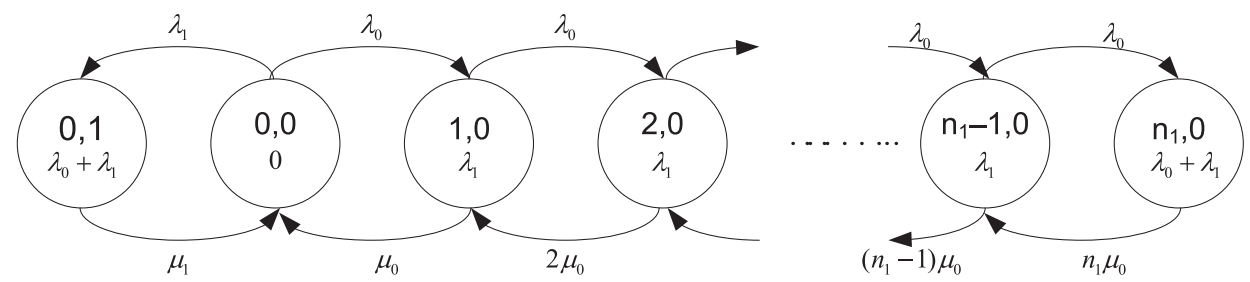

Fig. 4. The MMPP describing the overflow traffic from window- 1 to window- 2 .

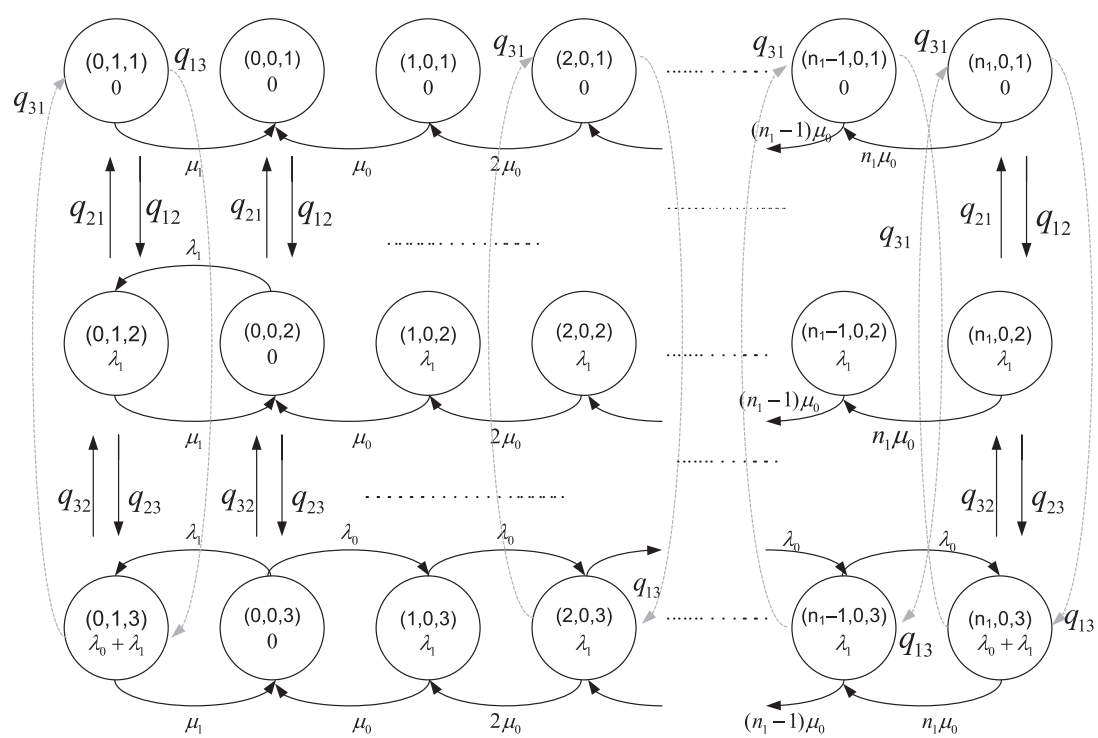

Fig. 5. The MMPP describing the overflow traffic from window- $i$ to window- $(i+1)$. When $i=H$, this MMPP characterizes the traffic that is blocked.

Due to notational burden, we avoid an elaborate description of the proposed algorithm for $K=3$. However, we briefly describe the extensions to Algorithm 1 needed to solve the $K=3$ case. For $K=3$, each of the $H=m_{2}$ windows can be fully occupied by one class- 2 demand or a number of class0 and class- 1 demands. For each window, there are actually $m_{1}$ sub-windows each of which can be occupied by just one class- 1 demand or up to $n_{1}$ class- 0 demands. Therefore, the CTMC which arises in Step 6 of Algorithm 1 would comprise the states $\left(i_{0}^{j}, i_{1}^{j}, l\right): 0 \leq i_{0}^{j} \leq n_{1}, i_{1}^{j}=0,1,1 \leq j \leq$ $m_{1}, 1 \leq l \leq 4 G$ for the case $K=3$ since in this case there would be $I=4$ distinct rates, namely $0, \lambda_{2}$, and $\lambda_{1}+\lambda_{2}$, and $\lambda_{0}+\lambda_{1}+\lambda_{2}$, and one additional state indicating that the window is occupied by a single class- 2 connection. Moreover, $i_{0}^{j}\left(i_{1}^{j}\right)$ keeps track of the number of class-0 (class- 1 ) demands in use in sub-window $j$ when starting to count from the low end of the window. Regarding computational complexity, following similar arguments used for the case $K=2$, one can conclude that at each step of analysis (except for the first step) the steady-state solution of a CTMC with $4 G\left(\left(m_{0}+2\right)^{m_{1}}+1\right)$ states needs to be obtained yielding an overall computational complexity of $\mathcal{O}\left(G^{3} m_{2} m_{0}^{3 m_{1}}\right)$. In this study, we have only implemented the proposed algorithm for $K \leq 3$, but the proposed algorithm can also be extended to more than three traffic classes at the expense of increased computational complexity, which is left outside the scope of this paper.

\section{Numerical results}

We use the proposed analytical method to numerically study the blocking performance of several two- and threeclass aligned EOLs and also validate the proposed method using simulations. In the numerical examples to follow, we assume $\mu_{k}=1$, for $k=0, \ldots, K-1$, i.e., average holding times for all traffic classes are the same. As stated before, we employ the EI and EL traffic mixture models. When $K=2$, we consider the two demand size vectors $\mathbf{n}=(1,4)$ and $\mathbf{n}=$ $(1,8)$. For $K=3$, we study $\mathbf{n}=(1,2,4)$ and $\mathbf{n}=(1,4,8)$ all of which are reasonable choices for EONs [10]. The proposed analytical method is implemented with 64-bit Matlab 8.1 running on a Windows 7 laptop which has an Intel i5 $460 \mathrm{M}$ processor with a core speed of $2.53 \mathrm{GHz}$ and $4 \mathrm{~GB}$ of RAM.

\subsection{Example 1}

The proposed analytical method is employed to obtain the per-class blocking probabilities in a three-class EOL with normalized load $\rho=0.7$ with demand size vector $\mathbf{n}=(1,4,8)$ in Fig. 6 for two values of the parameter $G$ and for both EI and EL traffic mixture scenarios and as a function of the EOL capacity $N$. The blocking probabilities obtained with [20] in the absence of contiguous allocation constraint, i.e., non-contiguous allocation, is also depicted as well as the 


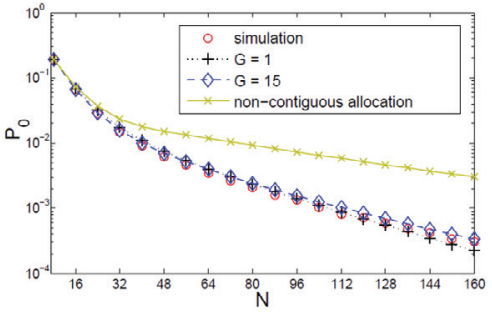

(a) $P_{0}$ for EI mixture

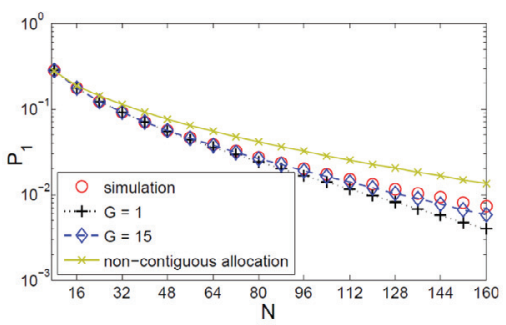

(c) $P_{1}$ for EI mixture

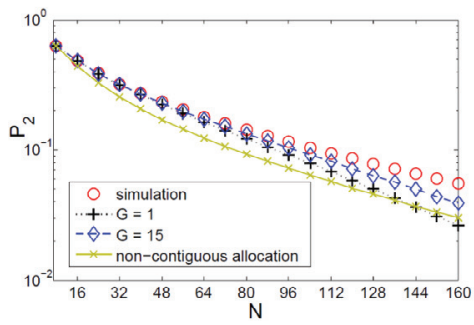

(e) $P_{2}$ for EI mixture

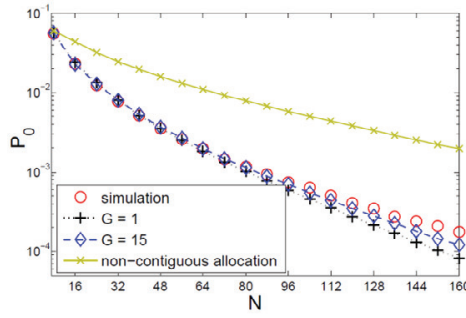

(b) $P_{0}$ for EL mixture

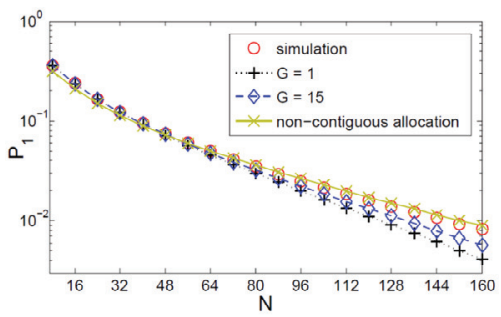

(d) $P_{1}$ for EL mixture

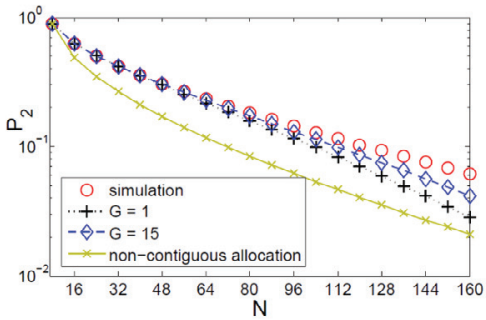

(f) $P_{2}$ for EL mixture

Fig. 6. The per-class blocking probabilities $P_{i}, i=0,1,2$, when $\mathbf{n}=(1,4,8), \rho=0.7$, as a function of link capacity $N$ obtained with simulations, the proposed method with two choices of the parameter $G=1,15$, and the algorithm in [20] without contiguous allocation constraints.

simulation results. All the per-class blocking probabilities are captured well especially with the choice of $G=15$ for relatively low values of $N$ when compared with the $G=1$ case. When $N$ is such that $H=N / n_{2}=m_{2} \leq 10$ MMPP order reductions are performed, the per-class blocking probabilities are very well captured. However, each further MMPP order reduction procedure introduces additional errors and the accuracy is slightly compromised. It is clear that the order of the reduced order MMPP used at each step of the proposed algorithm is $4 G=60$ for the choice of $G=15$ and for improved accuracy for much larger values of $N$, increasing the order of the reduced order model would be necessary. We also observe that the blocking probabilities obtained in the presence and absence of the contiguity constraint are quite different. To explain this, in non-contiguous allocation, the fragmentation problem vanishes. Therefore, more class-2 demands are accepted with non-contiguous allocation in comparison with contiguous allocation since larger demands are the ones that are affected adversely from fragmentation. However, when more class- 2 demands are admitted into the EOL, the link becomes more loaded leaving less room for class- 0 and class- 1 demands, increasing their blocking probabilities with noncontiguous allocation. Moreover, we observe that blocking probabilities decrease with increased capacity $N$ under the same normalized load which is expected due to multiplexing gains.

\subsection{Example 2}

In this example, we study a dimensioning problem using the proposed analytical method. In particular, we address the problem of finding the minimum system capacity $N$ in an EOL so as to attain a desired Quality of Service (QoS) given the traffic intensities $\lambda_{k}, k=0, \ldots, K-1$. In a 3 -class system, since the blocking probability of class- 2 demands would be larger than that of the other classes, we set the QoS requirement to $P_{2}<10^{-\alpha}$ for a predetermined QoS parameter $\alpha$. In the same spirit, the QoS requirement for a 2-class system is set to $P_{1}<10^{-\alpha}$. In this example, we study the $\alpha=1$ and $\alpha=2$ cases. In Fig. 7, the number of windows necessary to attain the QoS requirement in four different scenarios involving two different values of $K$ and the two EL and EI traffic mixtures. Note that the system capacity $N$ would then be obtained by multiplying the number of windows by $n_{K-1}$. Both analysis and simulation results are reported in Fig. 7 with the analytical model using the parameter $G=15$. The analytical results match with simulations in all cases we studied with an error of at most $9 \%$. Therefore, we conclude that the 


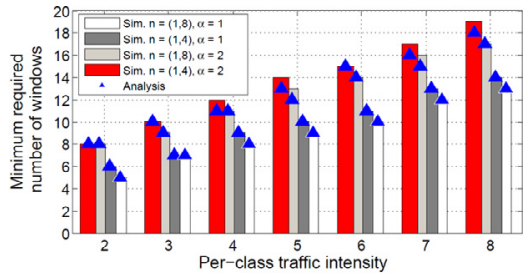

(a) $K=2$ and EL mixture

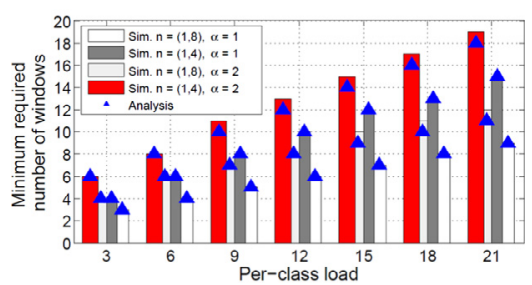

(c) $K=2$ and EI mixture

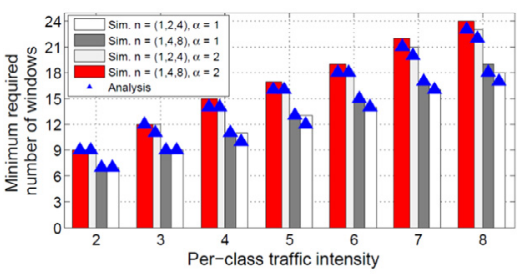

(b) $K=3$ and EL mixture

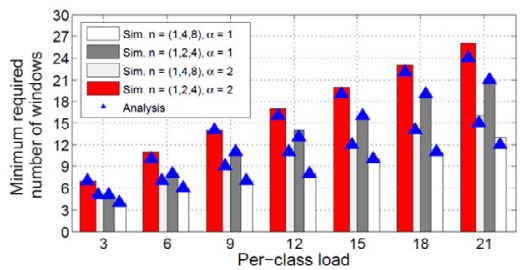

(d) $K=3$ and EI mixture

Fig. 7. The minimum required number of windows to ensure $P_{K-1}<10^{-\alpha}$ for $\alpha=1$ and 2 . The demand size vectors $\mathbf{n}=(1,4),(1,8),(1,2,4)$, and $(1,4,8)$ are studied in EL and EI mixture scenarios.

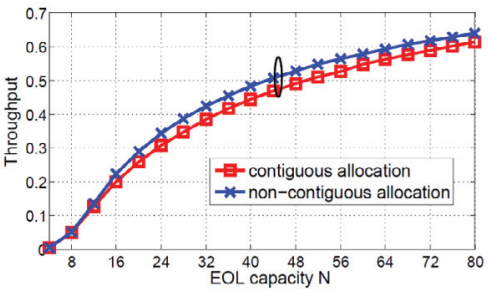

(a) $\mathbf{n}=(1,4)$ and EL mixture

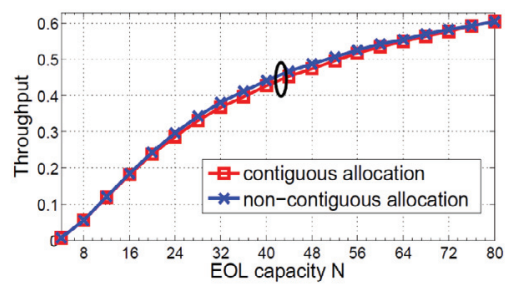

(c) $\mathbf{n}=(1,4)$ and EI mixture

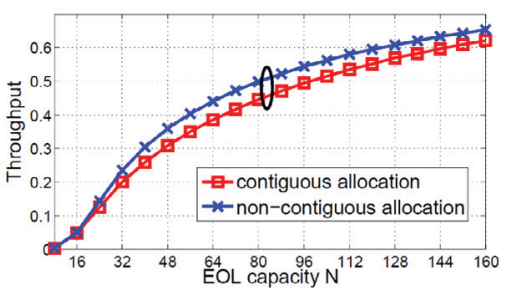

(b) $\mathbf{n}=(1,4,8)$ and EL mixture

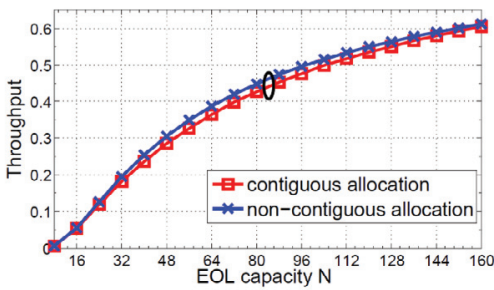

(d) $\mathbf{n}=(1,4,8)$ and EI mixture

Fig. 8. Throughput $T$ of an aligned EOL as a function of system capacity $N$ in comparison with that of a multi-server multi-class system with non-contiguous allocation for four different scenarios.

proposed analytical model can be used effectively for dimensioning purposes.

\subsection{Example 3}

We define the throughput $T$ obtainable at an EOL as the maximum normalized load at which a certain QoS is attained. In particular, we use the QoS requirement $P_{K-1}<$ 0.01 . Naturally, the throughput $T$ depends on the specific traffic mixture. Consequently, we study both EI and EL mixtures in this example by the analytical model only. Moreover, we also calculate the throughput $T$ when the contiguous allocation constraint is absent. Fig. 8 illustrates the throughput values as a function of the system capacity $N$ when the contiguous allocation constraint is present and absent for four different scenarios. We have the following observations:
- Throughput is always higher when the contiguous allocation constraint is absent in comparison with the case when the constraint is present. We define the contiguity penalty as the difference in these two throughput values. This penalty appears to be more substantial in the EL scenario in comparison with EI since in the latter, the overall EOL load is dominated by class- $(K-1)$ traffic which consequently reduces fragmentation stemming from contention of multiple traffic classes for spectral resources.

- Our findings demonstrate that the contiguity penalty is reduced as $N$ increases beyond a certain value and the penalty is larger for the $K=3$ case as opposed to $K=2$. This observation is in line with existing simulation results that EOL fragmentation increases with increased number of traffic classes [17]. 


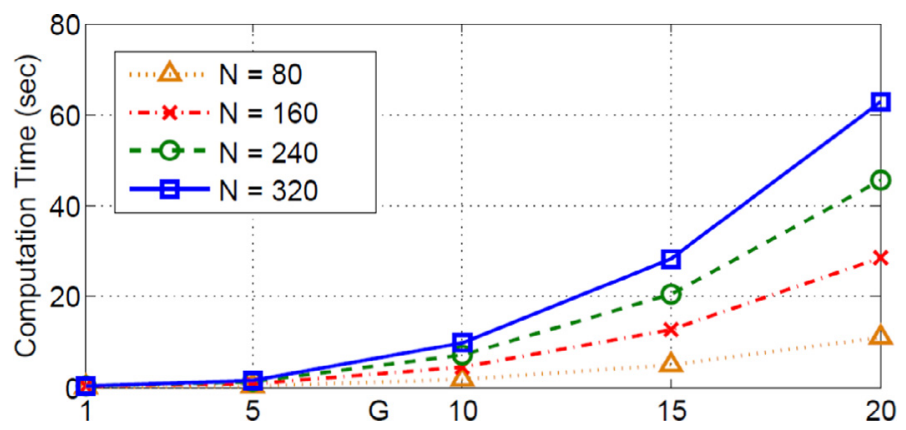

Fig. 9. The computation times for $\rho=0.7, \mathbf{n}=(4,8,16)$ for the EL traffic mixture case as a function of the order reduction parameter $G$ and for various values of the system capacity $N$.

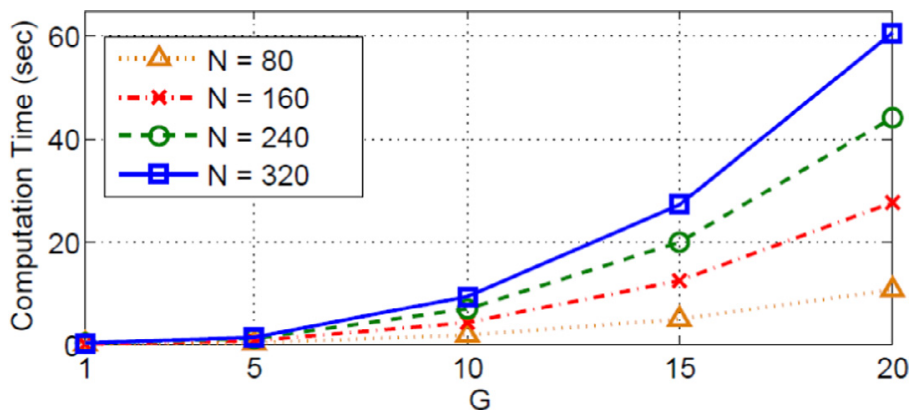

Fig. 10. The computation times for $\rho=0.7, \mathbf{n}=(4,8,16)$ for the El traffic mixture case as a function of the order reduction parameter $G$ and for various values of the system capacity $N$.

- Throughput increases monotonically and the contiguity penalty appears to shrink with increasing system capacity $N$. Based on these observations, we tend to believe that EOLs can be operated at high throughputs if the system capacity is selected to be far larger than the largest demand size $n_{K-1}$.

\subsection{Example 4}

In the final example, we provide the computation times required to run the analytical method for the case when $\rho=0.7$ and the demand size vector $\mathbf{n}=(4,8,16)$. Computation times are obtained by means of running the method ten times and then obtaining the average computation time out of these ten runs. We vary the order reduction parameter $G$ and the EOL capacity $N$ and report in Figs. 9 and 10 the computation times for the EL and EI traffic mixture scenarios, respectively. Note that the $\mathbf{n}=(4,8,16)$ aligned $E O L$ case with system capacity $N$ is statistically equivalent to the $\mathbf{n}=(1,2,4)$ case with capacity $N / 4$. Our numerical findings are in line with the computational complexity of the proposed method being $\mathcal{O}\left(G^{3} m_{2}\right)$ or equivalently $\mathcal{O}\left(G^{3} N\right)$, when the demand size vector $\mathbf{n}$ is fixed. We observe that problems satisfying the assumptions (a) and (b) can be approximately solved with computation times being far less than what would be required with simulations especially for cases associated with relatively low blocking probabilities. We also observe that the computation times for the EI and EL mixture cases are quite similar.

\section{Conclusions}

We study the blocking performance of a multi-class elastic optical link with the contiguous allocation constraint. We first propose a non-work-conserving spectrum allocation policy based on alignment and we show that alignment outperforms the conventional first fit-based work-conserving allocation policy without alignment, in terms of bandwidth blocking probability. We then propose a novel and systematic order reduction procedure for MMPPs and use this procedure as the numerical engine to approximately obtain the blocking probabilities in an aligned elastic optical link with up to three different traffic classes under certain demand size vector assumptions. The proposed stochastic model is numerically efficient and provides accurate estimates of the required system capacity to attain a certain level of QoS, making it amenable to be used for dimensioning purposes. We also introduce a new concept, called contiguity penalty, which is defined as the reduction in throughput at an EOL due to the contiguous allocation constraint. We show by analysis that the contiguity penalty tends to reduce when the system capacity is chosen large enough when alignment is employed for spectrum allocation. Removal of the assumptions on the demand size vector so as to handle other realistic scenarios and obtaining blocking probabilities accurately for very large system capacities are left for future research. Another potential future work is to extend the analysis of one single link to the performance modeling of a network of links. 


\section{References}

[1] E. Pincemin, Challenges of 40/100 Gbps deployments in long-haul transport networks on existing fibre and system infrastructure, in: Optical Fiber Communication Conference (OFC), 2010, pp. 1-3.

[2] O. Gerstel, M. Jinno, A. Lord, S.J.B. Yoo, Elastic optical networking: a new dawn for the optical layer? IEEE Commun. Mag. 50 (2) (2012) 12-20.

[3] M. Jinno, H. Takara, B. Kozicki, Y. Tsukishima, Y. Sone, S. Matsuoka, Spectrum-efficient and scalable elastic optical path network: architecture, benefits, and enabling technologies, IEEE Commun. Mag. 47 (11) (2009) 66-73.

[4] P. Gambini, M. Renaud, C. Guillemot, F. Callegati, I. Andonovic, B. Bostica, D. Chiaroni, G. Corazza, S.L. Danielsen, P. Gravey, P.B. Hansen, M. Henry, C. Janz, A. Kloch, R. Krahenbuhl, C. Raffaelli, M. Schilling, A. Talneau, L. Zucchelli, Transparent optical packet switching: network architecture and demonstrators in the KEOPS project, IEEE J. Sel. Areas Commun. 16 (1998) 1245-1259.

[5] C. Qiao, M. Yoo, Optical burst switching (OBS) - a new paradigm for an optical Internet, J. High Speed Netw. (JHSN) 8 (1) (1999) 69-84.

[6] L. Xu, H. Perros, G. Rouskas, Techniques for optical packet switching and optical burst switching, IEEE Commun. Mag. 39 (1) (2001) 136-142.

[7] L. Velasco, M. Klinkowski, M. Ruiz, J. Comellas, Modeling the routing and spectrum allocation problem for flexgrid optical networks, Photonic Netw. Commun. 24 (3) (2012) 177-186.

[8] P. Wright, A. Lord, L. Velasco, The network capacity benefits of Flexgrid, in: 17th International Conference on Optical Network Design and Modeling (ONDM), 2013, pp. 7-12.

[9] G. Zhang, M. De Leenheer, A. Morea, B. Mukherjee, A survey on OFDMbased elastic core optical networking, IEEE Commun. Surv. Tutorials 15 (1) (2013) 65-87.

[10] A. Castro, L. Velasco, M. Ruiz, M. Klinkowski, J.P. Fernández-Palacios, D. Careglio, Dynamic routing and spectrum (re) allocation in future flexgrid optical networks, Comput. Netw. 56 (12) (2012) 2869-2883.

[11] K. Christodoulopoulos, I. Tomkos, E. Varvarigos, Routing and spectrum allocation in OFDM-based optical networks with elastic bandwidth allocation, in: Global Telecommunications Conference (GLOBECOM), 2010, pp. 1-6.

[12] R. Wang B. Mukherjee, Spectrum management in heterogeneous bandwidth optical networks, Opt. Switching Netw. 11, Part A (2014) 83-91.

[13] Y. Wang, J. Zhang, Y. Zhao, J. Liu, W. Gu, Spectrum consecutiveness based routing and spectrum allocation in flexible bandwidth networks, Chin. Opt. Lett. (2012) S106061-4.

[14] M. Jinno, B. Kozicki, H. Takara, A. Watanabe, Y. Sone, T. Tanaka, A. Hirano, Distance-adaptive spectrum resource allocation in spectrumsliced elastic optical path network, IEEE Commun. Mag. 48 (8) (2010) $138-145$.

[15] N. Sambo, F. Cugini, G. Bottari, G. Bruno, P. Iovanna, P. Castoldi, Lightpath provisioning in wavelength switched optical networks with flexible grid, in: 37th European Conference and Exhibition on Optical Communication (ECOC), 2011, pp. 1-3.

[16] K. Song, J. Zhang, Y. Zhao, X. Yu, Y. Yu, B. Chen, H. Yang, Service-oriented spectrum assignment algorithms in flexible bandwidth optical networks, in: 2012 Asia Communications and Photonics Conference (ACP), 2012, pp. 1-3.

[17] R. Yumer, N. Akar, E. Karasan, Class-based first-fit spectrum allocation with fragmentation avoidance for dynamic flexgrid optical networks, Opt. Switching Netw. 15 (2015) 44-52.

[18] J. Kaufman, Blocking in a shared resource environment, IEEE Trans. Commun. 29 (10) (1981) 1474-1481

[19] J.W. Roberts, A service system with heterogeneous user requirements: application to multi-service telecommunications, in: G. Pujolle (Ed.) Proceedings of Performance of Data Communications Systems and Their Applications, Amsterdam, Holland, 1981, pp. 423-431.

[20] G.L. Choudhury, K.K. Leung, W. Whitt, An algorithm to compute blocking probabilities in multi-rate multi-class multi-resource loss models, Adv. Appl. Prob. 27 (1995) 1104-1143.

[21] K.W. Ross, Multiservice Loss Models for Broadband Telecommunication Networks, Springer-Verlag New York, Inc., Secaucus, NJ, USA, 1995.

[22] Y. Yu, J. Zhang, Y. Zhao, X. Cao, X. Lin, W. Gu, The first single-link exact model for performance analysis of flexible grid WDM networks, in: Optical Fiber Communication Conference and Exposition and the National Fiber Optic Engineers Conference (OFC/NFOEC), 2013, pp. 1-3.
[23] Y. Yu, J. Zhang, Y. Zhao, H. Li, Y. Ji, W. Gu, Exact performance analytical model for spectrum allocation in flexible grid optical networks, Opt. Fiber Technol. 20 (2) (2014) 75-83.

[24] W. Shi, Z. Zhu, M. Zhang, N. Ansari, On the effect of bandwidth fragmentation on blocking probability in elastic optical networks, IEEE Trans Commun. 61 (7) (2013) 2970-2978.

[25] F. Paolucci, A. Castro, F. Fresi, M. Imran, A. Giorgetti, B. Bhownik, G. Berrettini, G. Meloni, F. Cugini, L. Velasco, L. Poti, P. Castoldi, Active PCE demonstration performing elastic operations and hitless defragmentation in flexible grid optical networks, Photonic Netw. Commun. 29 (1) (2015) 57-66.

[26] A. Castro, L. Velasco, M. Ruiz, J. Comellas, Single-path provisioning with multi-path recovery in flexgrid optical networks, in: 4th Internationa Congress on Ultra Modern Telecommunications and Control Systems and Workshops (ICUMT), 2012, pp. 745-751.

[27] ITU-T, Spectral grids for WDM applications: DWDM frequency grid, Recommendation G.694.1, International Telecommunication Union, 2012.

[28] W. Fischer, K. Meier-Hellstern, The Markov-modulated Poisson process (MMPP) cookbook, Perform. Eval. 18 (2) (1993) 149-171.

[29] A. Kuczura, The interrupted Poisson process as an overflow process, Bell Syst. Tech. J. 52 (1973) 437-448

[30] K.S. Meier-Hellstern, The analysis of a queue arising in overflow models, IEEE Trans. Commun. 37 (4) (1989) 367-372.

[31] L. Bright, P. Taylor, Calculating the equilibrium distribution in level dependent quasi-birth-and-death processes, Commun. Stat. Stochastic Models 11 (3) (1995) 497-525.

[32] J. Ye, S.-Q. Li, Folding algorithm: a computational method for finite QBD processes with level-dependent transitions, IEEE Trans. Commun. 42 (234) (1994) 625-639.

[33] G. Latouche, V. Ramaswami, Introduction to Matrix Analytic Methods in Stochastic Modeling, ASA-SIAM Series on Statistics and Applied Probability, Society for Industrial and Applied Mathematics (SIAM) Philadelphia, PA, USA, 1999.

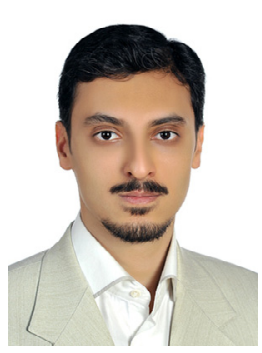

Kaveh Vaezi is currently a PhD student at Sharif University, Iran. He visited Bilkent University, Ankara, Turkey for a six months period September 2013-March 2014. His current research interests include performance modeling of computer and communication systems and networks.

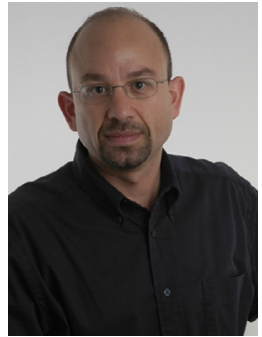

Nail Akar received the B.S. degree from Middle East Technical University, Turkey, in 1987 and M.S. and Ph.D. degrees from Bilkent University, Ankara, Turkey, in 1989 and 1994, respectively, all in electrical and electronics engineering. From 1994 to 1996 , he was a visiting scholar and a visiting assistant professor in the Computer Science Telecommunications program at the University of Missouri-Kansas City. He joined the Technology Planning and Integration group at Long Distance Division, Sprint, Overland Park, Kansas, in 1996, where he held a senior member of technical staff position from 1999 to 2000. Since 2000, he has been with Bilkent University, Turkey, currently as a professor at the Electrical and Electronics Engineering Department. He visited the School of Computing, University of Missouri-Kansas City, as a Fulbright scholar in 2010 for a period of six months. His current research interests include performance analysis of computer and communication systems and networks, performance evaluation tools and methodologies, design and engineering of optical and wireless networks, queuing systems, and resource management. 